\title{
Nonlinear Vibration of Beams and Rectangular Plates
}

By Joe G. Etsiey, Department of Aeronautical and Astronautical Engineering, University of Michigan, Ann Arbor, Michigan, USA

\section{Introduction}

The influence of initial membrane stress on the free and forced nonlinear vibration of beams and rectangular plates is investigated by means of a simple extension of the results for the unstressed case. A single mode representation is used; both simply supported and clamped beam and plate boundary conditions are considered; and the discussion includes the post buckling region.

\section{Notation}

$a, b, h$ plate width, length, and thickness, respectively

$r \quad a / b$, plate aspect ratio

$t \quad$ time

$u, v, w$ displacements in $x, y, z$ directions, respectively

$x, y$ coordinates in plane of plate

$D$ plate flexural rigidity

EI beam flexural rigidity

$F \quad$ stress function

@ mass density

v Poisson's ratio

Subscripts:

$s$ simply supported beam

c clamped beam

ss simply supported plate

cc clamped plate

Other symbols are defined in the text.

\section{Free Vibration of Beams}

When a beam with ends restrained to remain a fixed distance apart vibrates laterally there is stretching of the median line. This effect is usually accounted for in the strain-displacement relation by taking

$$
\varepsilon_{y}=v_{y}+\frac{1}{2}(w, y)^{2} .
$$


If the other usual assumptions of simple beam vibration theory are retained the following equation results for a beam of unit width

$$
\varrho h w_{t t}+\left(E I w_{y y}\right)_{y y}-\frac{E h}{b}\left[v_{0}+\frac{1}{2} \int_{0}^{b}\left(w_{y}\right)^{2} d y\right] w_{y_{y}}=P(y, t),
$$

where $v_{0}$ represents an initial axial displacement measured from the unstressed state.

This equation has been investigated by WoINowsKY-KRIEGER [1]1) and BuRGREEN [2] for a simply supported beam under the assumption of a single mode

$$
w(y, t)=b \xi(t) \sin \frac{\pi y}{b} .
$$

Application of GaLERKIn's method leads to the following equation of motion for free vibration

where

$$
\xi_{\tau_{\tau}}+p \xi+q \xi^{3}=0,
$$

$$
p=p_{s}=\frac{\pi^{4}}{12} \alpha^{2}\left(1-\lambda_{s}\right), \quad q=q_{s}=\frac{\pi^{4}}{4}, \quad \tau=\left(\frac{E}{\varrho}\right)^{1 / 2} \frac{t}{b}, \quad \alpha=\frac{h}{b} .
$$

The parameter $\lambda_{s}$ is a measure of the initial axial displacement and is defined

$$
\lambda_{s}=\frac{v_{0}}{v_{0_{c r}}},
$$

where $v_{0_{r}}$ is the axial displacement which produces the buckling load. Thus $\lambda_{s}>1$ refers to the post buckling region. It should be noted that Equation (2) and hence Equation (4) retains validity for $\lambda_{s}>1$. The exact solution to Equation (4), which may be obtained in terms of elliptic functions, is fully presented in Reference [2] and will not be repeated here.

A beam with both ends clamped may be investigated in a similar manner. However, one does not have so obvious a choice for an assumed mode since the linear vibration mode changes with axial load and in general is different from the buckling mode. As the axial load is increased in compression the mode shape for linear vibration more closely resembles the buckling mode and at buckling (for which the linear vibration frequency goes to zero) the two modes are identical. Thus

$$
w(y, t)=b \xi(t)\left(1-\cos \frac{2 \pi y}{b}\right)
$$

is a simple and logical choice. This leads to a slightly high value for the linear vibration frequency for axial compressive loads less than the buckling load.

The resulting equation of motion is of the same form as Equation (4) where now

$$
p=p_{c}=\frac{4 \pi^{4}}{q} \alpha^{2}\left(1-\lambda_{c}\right), \quad q=q_{c}=\frac{4 \pi^{4}}{3} .
$$

The parameter $\lambda_{c}$ is defined in the same way as $\lambda_{s}$ with $v_{0}$ now referred to a clamped beam. It is interesting to note that if it is assumed that

$$
\lambda_{c}=\lambda_{s}
$$

1) Numbers in brackets refer to References, page 174 . 
the equation for the clamped beam may be rewritten

$$
\xi_{, \tau \tau}+\frac{16}{3}\left(p_{s} \xi+q_{s} \xi^{3}\right)=0 .
$$

From this it may be concluded that, subject to the restrictions imposed by Equations (6) and (8), the ratio of the square of the nonlinear frequency of the clamped beam to that of the simply supported beam is always 16/3. Thus, if the frequency of one is known the other may be determined immediately. It should be noted that Equation (8) implies that the load on the clamped beam is four times the load on the simply supported beam, all other factors being equal.

\section{Free Vibration of Plates}

The effect of large deflection on the free vibration of rectangular plates has been investigated by $\mathrm{CHU}$ and HeRrmanN [3] for simply supported plates and by YamaKI [4] for simply supported and clamped plates. The dynamic von Kàrmàn equations, which are used, may be written

$$
\begin{aligned}
& \nabla^{4} F=E\left(w_{, x y}^{2}-w_{x x} w_{y y}\right), \\
& D \nabla^{4} w-h\left(F_{, y y} w_{x x}+F,_{x x} w_{y_{y}}-2 F,_{x y} w_{x y}\right)+\varrho h w_{, t}=P(x, y, t),
\end{aligned}
$$

where

$$
\sigma_{x}=F,_{y y}, \quad \sigma_{y}=F,_{x x}, \quad \tau_{x y}=-F,_{x y}
$$

are the membrane stresses. The single mode assumed for the simply supported plate is

$$
w(x, y, t)=b \xi(t) \sin \frac{\pi x}{a} \sin \frac{\pi y}{b}
$$

and for the clamped plate is

$$
w(x, y, t)=b \xi(t)\left(1-\cos \frac{2 \pi x}{a}\right)\left(1-\cos \frac{2 \pi y}{b}\right) .
$$

In Reference [3] the inplane boundary conditions are provided by edges restrained to remain a fixed distance apart. In Reference [4] these conditions as well as certain force free conditions are imposed. In each case an equation of the form of Equation (4) results when a single term Galerkin procedure is applied. These results may be extended to include the effects of initial edge displacement. The coefficients of Equation (4) for the simply supported plate are then

$$
p=p_{s s}=\frac{\pi^{4}}{12} \alpha^{2}\left(1-\lambda_{s s}\right)\left(\frac{1}{\gamma^{2}}+1\right)^{2}, \quad q=q_{s s}=\pi^{4}\left(\frac{3-v^{2}}{16}+\frac{v}{4 \gamma^{2}}+\frac{3-v^{2}}{16 \gamma^{4}}\right)
$$

and for the clamped plate are

$$
\begin{aligned}
& p=p_{c c}=\frac{4 \pi \pi^{4}}{27} \alpha^{2}\left(1-\lambda_{c c}\right)\left(3+\frac{2}{r^{2}}+\frac{3}{r^{4}}\right), \\
& q=q_{c c}=\pi^{4}\left[\frac{1}{8}\left(1+\frac{2 v}{\gamma^{2}}+\frac{1}{r^{4}}\right)\right. \\
& \left.+\frac{2}{9}\left(1-v^{2}\right)\left(\frac{17}{32}+\frac{17}{32 \gamma^{4}}+\frac{1}{\left(1+r^{2}\right)^{2}}+\frac{1}{4\left(1+4 r^{2}\right)^{2}}+\frac{1}{4\left(4+r^{2}\right)^{2}}\right)\right] .
\end{aligned}
$$


In addition the nondimensional time for a plate is

$$
\tau=\left[\frac{E}{\varrho\left(1-v^{2}\right)}\right]^{1 / 2} \frac{t}{b} .
$$

The parameters $\lambda_{s s}$ and $\lambda_{c c}$ are measures of the initial edge displacement. Consider, for example, edge displacements which, for values equal to and below the buckling values, produce uniform stresses on all four edges. Thus

$$
\sigma_{x}=\sigma_{y}=\frac{E}{(1-\nu)} \frac{u_{0}}{a}=\frac{E}{(1-\nu)} \frac{v_{0}}{b} .
$$

The parameter $\lambda$ is now defined

$$
\lambda=\frac{u_{0}}{u_{0_{c r}}}=\frac{v_{0}}{v_{0} c r},
$$

where for a simply supported plate

$$
\left(\frac{u_{0}}{a}\right)_{c r}=\left(\frac{v_{0}}{b}\right)_{c r}=-\frac{\pi^{2} \alpha^{2}}{12(1+v)}\left(\frac{1}{r^{2}}+1\right)
$$

and for a clamped plate

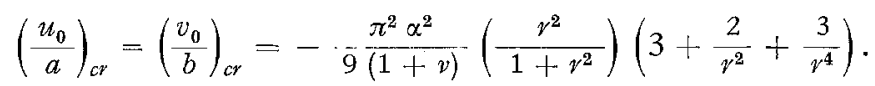

This latter value is obtained from an approximate solution based on a single term assumed mode of the form of Equation (12) and is known to give good accuracy. See Timoshenko and Gere [5].

If the uniform edge stress is considered to originate from the uniform heating of a plate with restrained edges then $\lambda$ may be identified as simply

$$
\lambda=\frac{\Delta T}{\Delta T_{e r}},
$$

where $\Delta T_{c r}$ is the buckling temperature.

\section{Forced Vibration - Harmonic}

Consider the forced vibration which occurs when a uniform load is applied periodically. Let $P(y, t)$ in Equation (2) and $P(x, y, t)$ in Equation (10) be of the form $B f(t)$ where $B$ is a constant and $f(t)$ is periodic. The equation of motion becomes

where $\beta$ is, respectively

$$
\xi,_{\tau \tau}+p \xi+q \xi^{3}=\beta f(\tau),
$$

$$
\beta_{s}=\frac{4 B}{\pi E \alpha}, \quad \beta_{c}=\frac{2 B}{3 E \alpha}, \quad \beta_{s s}=\frac{16\left(1-\nu^{2}\right) B}{\pi^{2} E \alpha}, \quad \beta_{c c}=\frac{4\left(1-\nu^{2}\right) B}{9 E \alpha} .
$$

This equation may be recognized as the familiar DUfFING's equation which is extensively discussed in books on nonlinear oscillation, as, for example, in SToker [6]. It is discussed in reference to the vibration of simply supported beams by METTLER [7] and in reference to plate vibration by YAMAKI [4] and LIN [8] for harmonic forcing

$$
f(\tau)=A \cos \omega \tau
$$


In each case a first order approximate steady state solution is obtained by assuming

$$
\xi(\tau)=A \cos \omega \tau
$$

which leads to the following frequency-amplitude relation (using, for example, the Ritz-Galerkin method)

$$
\omega^{2}=(p-\beta)+\frac{3}{4} q A^{2} .
$$

This result is valid for the cases considered here subject to certain limitations and interpretations imposed by the range of values and sign of the parameter $p$.

It is recognized that as $\lambda \rightarrow 1, p \rightarrow 0$, and when $\lambda>1, p<0$. Certain approximations often used in solving DUFFING's equation and in determining the stability of these solutions require that

$$
\frac{3}{4} \frac{q}{p} A^{2} \ll 1, \quad \frac{\beta}{p} \ll 1 .
$$

Clearly this may not be the case for the range of interest here and these approximations must be avoided.

The effects of varying the parameter $\lambda$ can best be illustrated by an example. The response of a simply supported beam with $\alpha=0.005$ has been computed from

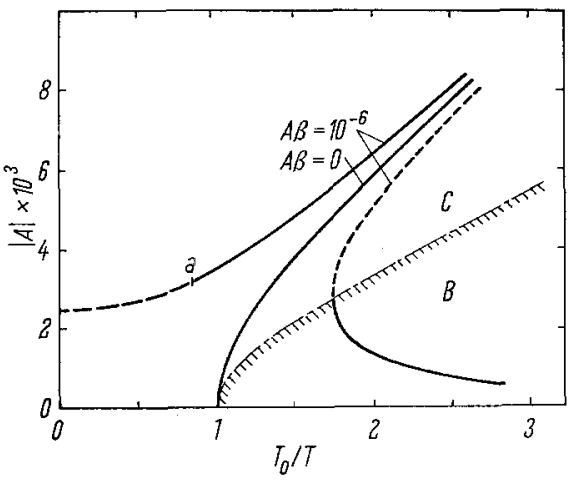

Figure la

Harmonic forcing, $\lambda=0$.

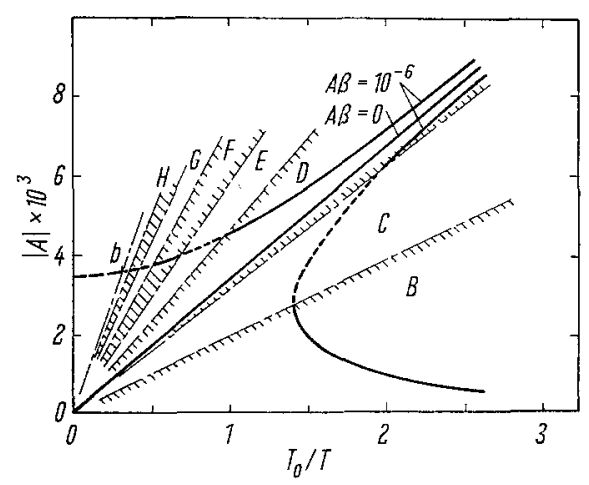

Figure 1b

Harmonic forcing, $\lambda=1$.

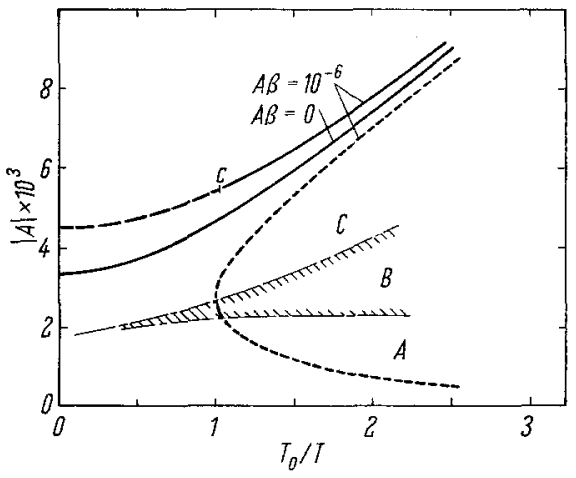

Figure 1c

Harmonic forcing, $\lambda=2$. 
Equation (24) for free and forced steady state oscillation. Values of $\lambda=0,1,2$ have been considered and typical results are plotted in Figures $1 \mathrm{a}, 1 \mathrm{~b}$, and $1 \mathrm{c}$. The ordinate is the absolute value of the amplitude $A$, and the abscissa is the ratio of the period of the linear vibration for $\lambda=0$ to the actual period.

To investigate the stability of these steady state solutions let

$$
\xi(\tau)=A \cos \omega \tau+\gamma(\tau),
$$

where $\gamma$ is a small variation. If Equation (26) is substituted into Equation (21) and only linear terms in $\gamma$ are retained, it follows that

$$
\gamma_{\tau \tau}+\left(p+3 q A^{2} \cos ^{2} \omega \tau\right) \gamma=0
$$

This is a form of HiLL's equation known as the Mathieu equation which may be put in a standard form

where

$$
\gamma_{z z}+(\delta+\varepsilon \cos z) \gamma=0
$$

$$
\delta=\frac{p+3 q A^{2} / 2}{4 \omega^{2}}, \quad \varepsilon=\frac{3 q A^{2}}{8 \omega^{2}}, \quad z=2 \omega \tau
$$

The exact solution of Equation (28) for the stability boundaries in terms of $\delta$ and $\varepsilon$ is discussed in Reference [6]. A portion of these boundaries are presented in Figure 2. The shaded regions are stable.

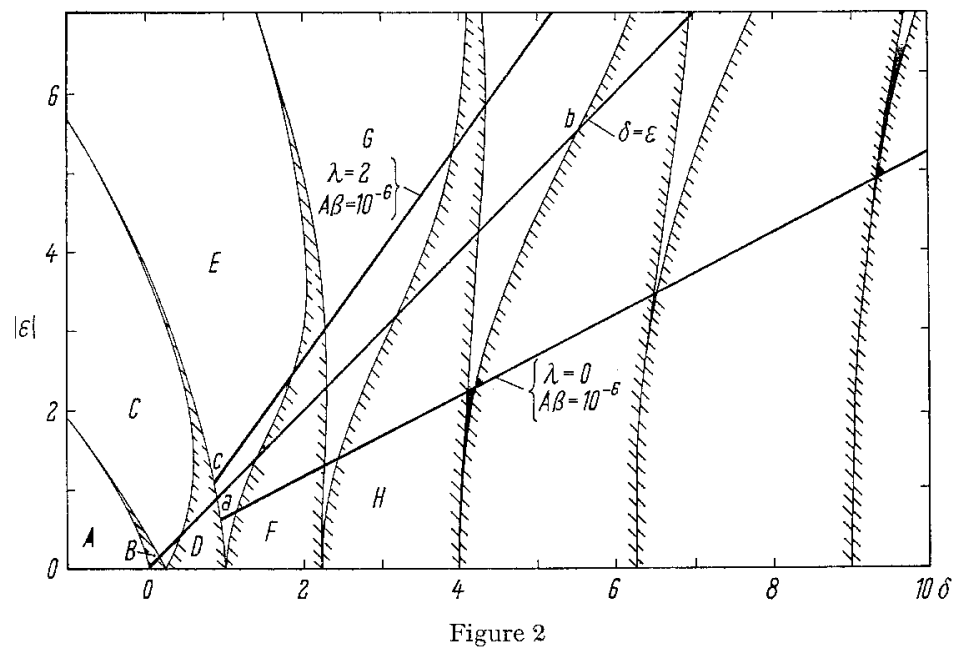

Stability boundaries for Mathieu equation.

The results for $\lambda=0$ are presented in Figure 1a. This case is identical to the problem of a mass on a hard spring and is the one considered for beams and plates in References [4], [7], and [8]. The upper branch of the response curve is for the most part stable. To the left of the point $a$ there are narrow regions of instability which may be identified in Figure 2 where a portion of this curve is replotted in terms of $\delta$ and $\varepsilon$. The upper portion of the lower branch is unstable. It lies in the region $C$ of Figure 2 . The lower portion of this branch is stable and lies in the region $B$ of Figure 2 . The 
boundary between regions $B$ and $C$ is reproduced in Figure 1a. As $\lambda$ is increased similar results will be obtained with the free vibration curve moving to the left and becoming straighter.

When $\lambda=1, p=0$, and the curves in Figure $1 \mathrm{~b}$ may be obtained. The free vibration curve is now a straight line. The regions of stability and instability are determined by the requirement $\delta=\varepsilon$ obtained from inspection of the coefficients of Equation (28). This condition is plotted in Figure 2 and the results are transferred to Figure 1b where the stability boundaries are seen to be radial lines. There are additional regions of stability and instability to the left of the point $b$ corresponding to larger values of $\delta$ and $\varepsilon$.

A further increase in $\lambda$ leads to still different results. There is now a minimum amplitude for which free vibration can occur which results from the form of the assumed solution in Equation (23). This solution requires symmetrical vibration about the flat position. For the buckled case $(\lambda>1)$ another type of motion is possible in which vibration takes place about the static buckled position on one side of the flat position. This latter type of motion is not considered here. Therefore, in the above it is assumed that the proper conditions exist for exciting the motion.

The curves for $\lambda=2$ are given in Figure 1c. To the left of the point $c$ the upper branch is for the most part unstable. A portion of this curve is replotted in Figure 2. The lower branch is unstable except for one narrow region of stability shown.

\section{Forced Vibration - Elliptic}

An exact solution of Equation (21) is possible if $f(\tau)$ is properly chosen. One possibility of interest is to choose an elliptic forcing function

$$
f(\tau)=A \operatorname{cn}(\omega \tau, k)
$$

for which Equation (21) has as its solution

$$
\xi(\tau)=A c n(\omega \tau, k)
$$

subject to the conditions

$$
\omega^{2}=p-\beta+q A^{2}, \quad k^{2}=\frac{q A^{2}}{2 \omega^{2}} .
$$

The period of the oscillation is given by

$$
T=\frac{4 k}{\omega}=\frac{4}{\omega} \int_{0}^{\pi / 2} \frac{d \varphi}{\left[1-k^{2} \sin ^{2} \varphi\right]^{1 / 2}} .
$$

This approach is of interest because it reduces to the exact solution for free vibration when $\beta=0$. It has been considered by LIN [8] for a simply supported plate with $\lambda=0$. The method is explained in greater detail by Hsu [9].

The response to elliptic forcing has been computed for the same example beam and the curves are plotted in Figures $3 \mathrm{a}, 3 \mathrm{~b}$, and $3 \mathrm{c}$. The stability of these solutions may be determined from the following equation

$$
\gamma_{{ }_{\tau \tau}}+\left(p+3 q A^{2} c n^{2}[\omega \tau, k]\right) \gamma=0 \text {. }
$$


This is a form of HILI's equation for which the general properties are discussed in Reference [6]. Presumably similar problems of stability would arise as in the case of harmonic forcing.

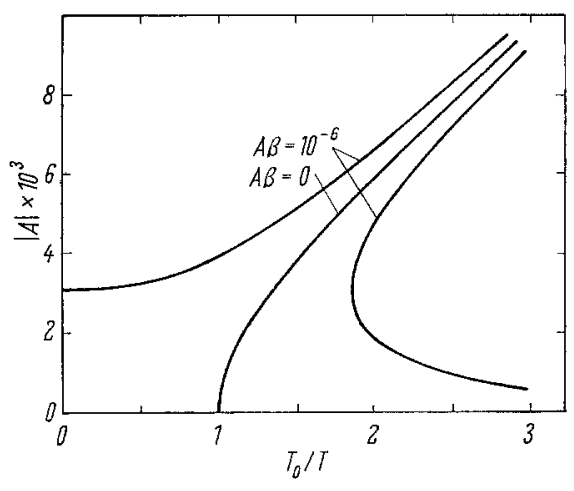

Figure $3 a$

Elliptic forcing, $\lambda=0$.

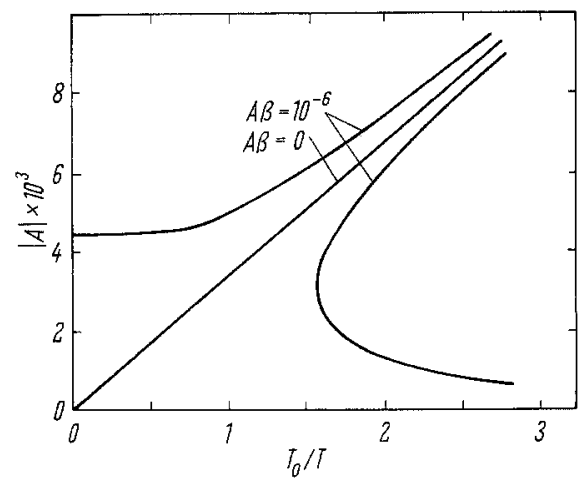

Figure $3 \mathrm{~b}$

Elliptic forcing, $\lambda=1$.

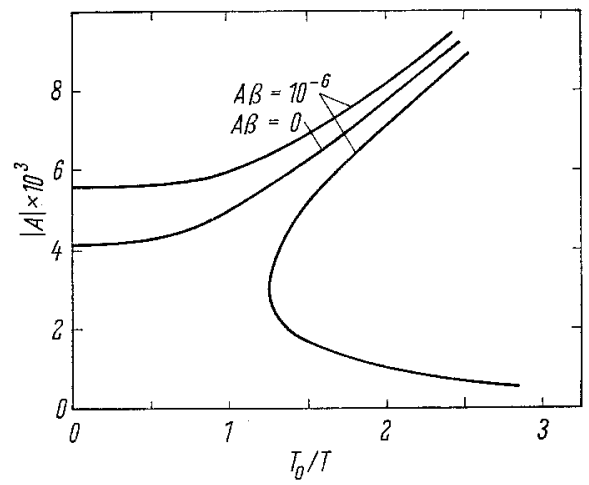

Figure 3c

Elliptic forcing, $\lambda=2$.

\section{REFERENCES}

[1] S. Woinowsky-Krieger, The Effect of Axial Force on the Vibration of Hinged Bars. J. appl. Mech. 17, 35 (1950).

[2] D. Burgreen, Free Vibrations of a Pin Ended Column with Constant Distance between Ends. J. appl. Mech. 18, 135 (1951).

[3] H. N. Chu and G. Herrmann, Influence of Large Amplitudes of Free Flexural Vibrations of Rectangular Elastic Plates. J. appl. Mech. 23, 532 (1956).

[4] N. Yamaki, Influence of Large Amplitudes on Flexural Vibrations of Elastic Plates. Z. angew. Math. Mech. 41, 501 (1961).

5] S. P. Trmoshenko and J. M. Gere, Theory of Elastic Stabitity (McGraw-Hill Book Co., Inc., 1961).

[6] J. J. Stoker, Nonlinear Vibrations (Interscience Publishers, Inc., 1950).

[7] E. Mettler, Zum Problem der Stabilität erzwungener Schwingungen elastischer Körper. Z. angew. Math. Mech. 37, 263 (1951). 
[8] Y. K. Lin, Response of a Nonlinear Flat Panel to Periodic and Randomly Varying Loadings. J. Aerospace Sci. 29, 1029 (1962).

[9] C. S. Hsu, On the Application of Elliptic Functions in Nonlinear Forced Oscillations. Quart. appl. Math. 17, 393 (1960).

\section{Zusammentassung}

Der Einfluss von Vorspannungen auf die freien und erzwungenen nichtlinearen Schwingungen von Balken und rechteckigen Platten wird mittels einer einfachen Erweiterung der Lösungen für Fälle ohne Vorspannung untersucht. Es wird eine einzige Koordinatenfunktion benützt; es werden einfach aufgelegte und eingespannte Fälle betrachtet; und die Diskussion wird auch auf den überkritischen Bereich ausgedehnt.

(Received: June 20, 1963.)

\section{Steady State Thermal Stresses in an Elastic Solid Bounded by Two Cones ${ }^{1}$ )}

By Subhendu K. Datta, Department of Mathematics, Rensselaer Polytechnic Institute, Troy, N.Y., USA

\section{Introduction}

In hydrodynamics similarity transformations have been used by many authors to reduce the number of independent variables in boundary layer equations. But their use in elasticity has not been explored very much. Mathematically speaking the similarity means a transformation of variables in a system of partial differential equations. BIRKHOFF [2] ${ }^{2}$ ) was the first to give a general method of obtaining similarity solutions of problems of hydrodynamics. Later MoRGAN [4] gave a rigorous theory using a one parameter group of transformations to reduce by one the number of independent variables in a system of partial differential equations. He also applied this to obtain the stress distributions within an elastic solid bounded by one or two cones [5]. For reference concerning the boundary layer equations. the reader may consult the bibliography given by AввотT and KLINE [1] at the end of their report, in which they give methods of finding similarity variables based upon the mathematical technique of solving partial differential equations by separation of variables. They discuss the solution of a problem of non-steady-state heat conduction in a semiinfinite rectangular corner in addition to getting the solutions for various fluid dynamic problems. Recently ManoHaR [3] gave a simple method of finding the similarity variables for a system of partial differential equations and applied this to some boundary layer problems. In this work we shall extend his method to include some thermo-elastic problems. Recently SingH [11] used Mellin transforms to solve a thermo-elastic problem for an elastic solid bounded by one or two cones. Muki and

1) This work has been done at the Mathematics Research Center, University of Wisconsin, under the U.S. Army Contract DA-11-022-ORD-2059.

$\left.{ }^{2}\right)$ Numbers in brackets refer to References, page $\mathbf{1 8 3}$. 